\title{
Behavioral activation for children and adolescents: a systematic review of progress and promise
}

\author{
Faith Martin ${ }^{1} \cdot$ Thomas Oliver $^{2}$
}

Received: 20 August 2017 / Accepted: 9 February 2018 / Published online: 23 February 2018

(c) The Author(s) 2018. This article is an open access publication

\begin{abstract}
Behavioral activation (BA) effectively treats depression in adults, and shows promise in treating anxiety. Research into its application to children and adolescents is emerging. This review aimed to explore the scope of studies, current evidence of effectiveness and how the intervention has been delivered and adapted, to inform future research. A systematic review was undertaken searching PsycInfo, PubMed including Medline, EMBASE, and Scopus for terms relating to BA and children and adolescents. Two researchers scored abstracts for inclusion. Data extraction was completed by one researcher and checked by another. 19 studies were identified, across 21 published articles. 12 were case studies, with three pre-post pilot designs and four randomized-controlled trials. Case studies found early support for the feasibility and potential effectiveness of BA to address both anxiety and depression. The RCTs reported largely positive outcomes. Meta-analysis of depression scores indicated that BA may be effective; however, high heterogeneity was observed. Sample sizes to date have been small. BA has been delivered by trained therapists, doctoral trainee psychologists, social workers, or psychology graduates. Studies are uniquely in high-income settings. Adaptations include flexibility in content delivery, youth friendly materials, and parental involvement. There is some limited evidence to support BA as effective for young people. Feasibility and acceptability are supported. Fully powered trials are now required, with expansion to delivery in low- and middle-income settings, and detailed consideration of implementation issues that consider culture and environment.
\end{abstract}

Keywords Behavioral activation $\cdot$ Depression $\cdot$ Anxiety $\cdot$ Children $\cdot$ Adolescents $\cdot$ Systematic review

\section{Introduction}

Adolescent mental health is currently recognized as a global priority [1]. Mental health problems most commonly emerge in adolescence, and treatment at this life-stage can prevent problems from being maintained into adulthood, to avoid life-long mental distress and the individual, social,

Electronic supplementary material The online version of this article (https://doi.org/10.1007/s00787-018-1126-z) contains supplementary material, which is available to authorized users.

Faith Martin

psmfm@bath.ac.uk

Thomas Oliver

thom.oliver@uwe.ac.uk

1 Department of Psychology, University of Bath, Claverton Down, Bath BA2 7AY, UK

2 University of the West of England, Frenchay Campus, Coldharbour Lane, Bristol BS16 1QY, UK and economic costs that accompany this [1]. Depression in young people is a leading cause of disease burden [2], as is anxiety [3]. Indeed, the two frequently co-occur amongst young people [4]. Other mental health and developmental disorders are responsible for a large proportion of disabilityadjusted life years lost amongst young people [2]. Effective, implementable interventions are then urgently required to address adolescent mental health. Behavioral activation has been found to be highly effective with adult depression [5, 6], yet its application with young people is in its infancy.

Cognitive-behavioral therapy (CBT) is recommended for treatment of children and adolescents [7, 8], along with interpersonal therapy, psychodynamic therapy, and family therapy [9]. However, access to treatment is low: in the UK, $55 \%$ of $12-15$ years with mental health problems receive no service for these needs [10]. Across Europe, access is estimated at around $26 \%$ of youth receiving mental health services within 12 months [11]. In low- and middle-income countries, there are often no or very few available services 
[12]. Increased access to treatment is an important goal, and interventions that can be implemented at scale are required.

\section{Relevance of BA to children and young people}

When considering appropriate interventions for child and young people to address their mental health difficulties, four issues must be considered:

1. The intervention is developmentally appropriate: for instance, avoiding complex abstract thinking [13].

2. Clinically, the intervention can address both depression and anxiety disorders, owing to high co-morbidities [4], and potentially relevant to a range of other difficulties to maximise impact.

3. Culturally sensitivity of the intervention to values and conceptualisations of distress to be able to be implemented widely, in diverse settings and in multi-cultural contexts [14].

4. Scaleability of the intervention, with minimal resources to allow wide implementation, including non-specialist delivery [15].

Behavioural activation (BA) fits these requirements being feasible for adolescents owing to its simple, behavioural, concrete focus. It has an emerging evidence base for the effectiveness of trans-diagnostic use [5, 16]. It is individualised and culturally sensitive [17] and has been delivered by non-specialists [18]. It is an effective treatment for adult depression, being cheaper to deliver but not clinically inferior to traditional CBT [6]. As such, BA has significant promise for treatment of young people. Research into BA for children and adolescents is an emerging field.

\section{Overview of behavioural activation}

BA originated as a behavioral treatment for depression, addressing the lack of positive reinforcement and excess of avoidance behaviors [19]. There are essentially two BA approaches in depression treatment: Washington BA [20] and brief BA treatment for depression or "BATD" [21]. The former focuses on the environmental context and how ineffective coping maintains depression [20]: depressed behaviors are seen as a way to cope, largely through avoidance, which is then directly addressed by engaging in helpful, alternative coping behaviors. It, therefore, requires a functional analysis of behaviors and tends to include a broader range of techniques than BATD. BATD seeks to increase reinforcement for non-depressive behaviors and decrease reinforcement for depression behaviors (such as avoidance) [21], however, does not require detailed functional analysis, and has emphasized the importance of the value placed by the individual of different behaviors.
Practically, the two types of BA share many features: both are based on the fundamental premise that behavioral avoidance is central to depression and both aim to increase adaptive activities that reduce depression, decrease activities that maintain depression, and problem solve barriers to reward [22]. Both forms of BA have core techniques of self-monitoring and activity scheduling [5]. Eight techniques have been identified as components of BA: activity monitoring, activity scheduling, contingency management, values, and goal assessment, skill training such as problem solving, relaxation, targeting verbal behaviors, and targeting avoidance [19].

\section{Defining BA when applied beyond depression}

BA was specifically designed to address depression. The extent to which behavioral therapy for non-depression problems can be called BA is unclear, rather than standard behavioral therapy. BA has increasingly been discussed in relation with anxiety disorders [23, 24]. Depression and anxiety share similarities, with negative reinforcement and avoidance in common [25]; therefore, BA may be applied in anxiety disorders. Classic behavioral therapy for anxiety often uses exposure-based classical conditioning theory to extinguish anxiety responses, whereas BA rests on operant conditioning principles and a functional contextualized perspective to address reinforcement [5]; therefore, the underpinning logic can define what is and is not $\mathrm{BA}$ in relation with anxiety.

For interventions, seeking to address problematic behaviors, in autism, for example, the picture may be less clear, as some behavioral therapy interventions use operant conditioning principles to shape behaviors, reinforcing nonproblematic behaviors [for example, 26]. This differs from $\mathrm{BA}$, as the behavioral shaping is completed for its own end, rather than to change behavior as a mechanism to influence mood state and psychological outcome [19]. For the purposes of this review, behavioral therapies using exposure only, or designed with the specific goal of altering undesired behaviors are not conceptualized as BA.

\section{Objectives of review}

A recently published systematic review with meta-analysis by Tindall and colleagues [27] focused on the effectiveness of BA to treat adolescent depression. The review reports that BA may be effective in the treatment of depression in young people; however, caution is required given a small number of studies and their methodological limitations. The review focuses solely on the treatment of depression, for which BA was originally designed. However, BA has been applied to other conditions with adults and the presence of such developments for treating young people is not yet reviewed. 
It is important to summarise the progress of BA as applied to young people, to help direct future research globally. Summarizing and analysing the details of what has been delivered, to whom, where, and how may guide researchers in the ongoing development of the intervention. A detailed review is required not only in relation with the potential effectiveness of the intervention, but also for how the intervention can be delivered and implemented, which should be considered early in the intervention development process [28, 29]. As such, this review seeks to extend beyond the scope Tindall and colleagues paper [27], with wider objectives.

The first objective of this review is to analyses the state of the science in relation with empirical basis and evidence base of BA as a treatment for children and young people, specifically to review studies reporting any quantitative data from children or adolescents who have undergone behavioral activation to provide initial analysis of outcomes on psychological wellbeing (including depression and anxiety), regardless of comparison group or study design, and the current reach and scope of the research. This includes investigating.

1. The study designs used.

2. The types of difficulties BA has been used for with children and young people.

3. The reach of the research to date in terms of participant characteristics, particularly in terms of cultural setting and ethnicity.

4. Evidence to date of effectiveness and/or adverse effects on depression and anxiety outcomes in children or adolescents.

The second objective is to explore how BA has been delivered to children and young people, including the location of delivery, therapist type and training, mode of delivery and number of sessions.

The third objective is to identify what has been learnt about adapting BA for children and young people, particularly in relation with parental involvement, and offer recommendations for future research. These objectives are essential to analyze knowledge to support future development of the intervention and its implementation.

\section{Methods}

There is no published review protocol; details of the methodology are provided here.

\section{Eligibility criteria}

Relating to study aims and as research into BA with children and young people is in its early stages, this review sought to identify all papers that had explicitly delivered a BA focused intervention, regardless of the study design, presence of comparison groups, length of follow-up, or the details of the psychological difficulty. Inclusion limits were: published papers with participants must include those up to the age of 19 years, based on the World Health Organization definition of "adolescence" [30], and interventions must use BA but not as part of a broader cognitive-behavioral or other therapeutic approach. Exclusion criteria were interventions which used behavioral therapy to reduce problematic behaviors only, prevention interventions, and purely qualitative papers.

\section{Information sources}

PsycInfo, PubMed including Medline, EMBASE, and Scopus were searched. No limits relating to date or language were included. Search was complete up to the end of the 1st week of December 2017. References included in studies identified as relevant were also searched for potentially relevant papers.

\section{Search}

Search terms were "Behavioral activation", "behavior therapy" or "behavioral therapy" (also capturing UK spelling), AND child, children, adolescent, adolescence, young person, young people, youth, teen, or teenager, with search terms in title or abstract only. This highly sensitive search was necessary to ensure all behavioral therapy interventions, that may include behavioral activation interventions, were captured.

\section{Study selection}

Duplicate records were removed. Abstracts of all identified studies were screened for eligibility by two researchers. Results were compared and final inclusions at abstract stage made. Differences in decisions to include/exclude were noted and discussed until agreement reached. Full texts for all included abstracts were sought. Full texts were inspected by one researcher, who proposed exclusion of the studies, where no relevant from the results could be found or where the intervention's full description revealed it to be not BA. The second researcher checked these decisions.

\section{Data collection process}

Full texts were inspected by one researcher, with data extracted into a prepared form. A second researcher checked one-third of the data extraction reports for accuracy and completeness (picked at random from the database). Errors or omissions were noted, and if differences were noted in two or more checked papers, it was planned to check data 
extraction for all included studies. As few additions or edits were made, no further checking was undertaken.

\section{Data items}

Data were captured covering details of the sample (including sample size, participant ages, where drawn from, psychological difficulties assessed), intervention description (covering general description and inclusion of listed identified BA technical content, for example, functional analysis, with an option for "other" content not included in our list), study design, results relating to psychological outcomes, and any information relating to the adaptation of BA for use with children and young people.

\section{Risk of bias in individual studies}

The Cochrane Collaboration's tool for assessing risk of bias (CCAR) at an individual study level was used for the RCTs only [31, 32].

\section{Summary measures}

Meta-analysis was conducted with data reported in randomized-controlled trials (RCT). Where multiple scales for the same outcome (e.g., a number of depression measures) were reported, measures shared between studies were selected where possible. The main summary measure is standardized mean difference, with $95 \%$ confidence intervals (CI).

\section{Synthesis of results}

A random effects model was used to synthesize results, owing to high expected (and observed) heterogeneity. Heterogeneity was assessed with the calculation of $I^{2}$ (value of $75 \%$ or more seen as "high" [31]).

\section{Risk of bias across studies}

A summary of risk of bias across studies was made based on the CCAR, as above.
Fig. 1 Screening and inclusion process for identified studies

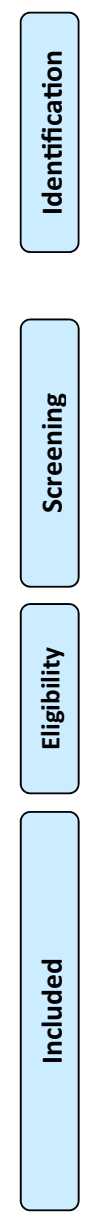

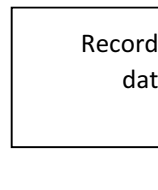

ords identified through abase searching $n=14964$

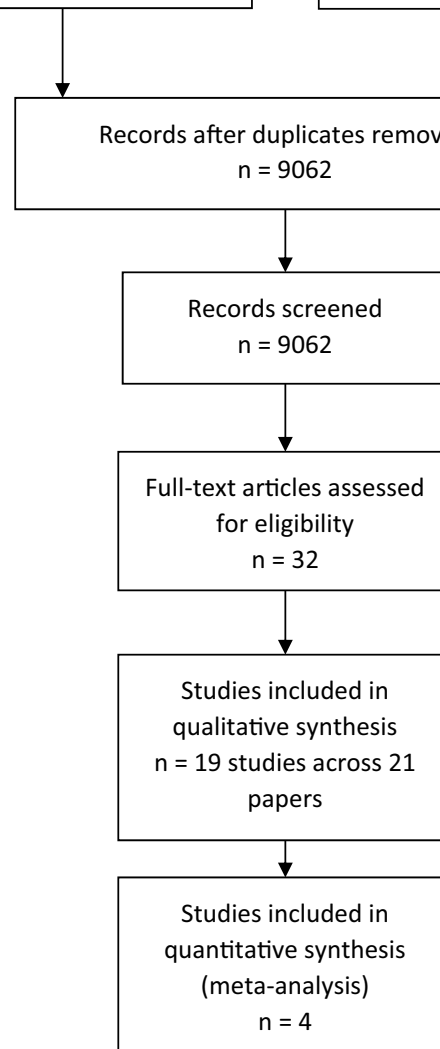

Additional records identified through other sources $\mathrm{n}=0$

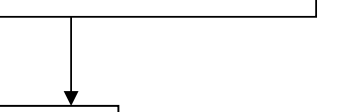

d

(1)

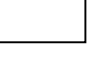
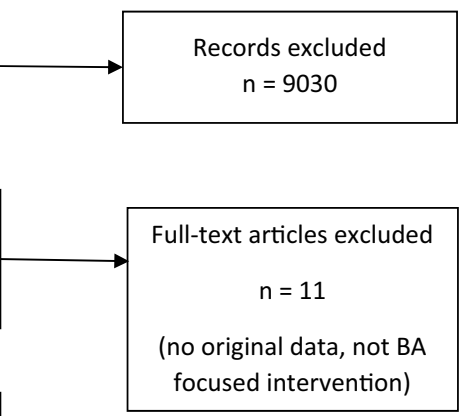

o original data, not BA 


\section{Results}

The flow of studies through the review is illustrated in Fig. 1, in accordance with reporting guidelines for systematic reviews [33].

\section{Study selection}

Agreement was achieved between the two researchers examining abstracts for inclusion/exclusion on 8360/8531 (98\%) abstracts and $100 \%$ agreement was achieved at full-text stage. 19 relevant studies, published across 21 papers, were included in the review.

Ten articles were excluded at full-text stage, as no original data were provided [34-37], all participants were over 18 years [38], or the intervention was not BA, being either broader cognitive-behavioral therapy or narrowing behavioral shaping [39-44].

Of note, two interventions describing themselves as BA were excluded. Both were with children with ADHD and sought to modify undesirable behaviors [41, 42]. It is difficult to distinguish whether these studies are truly BA, or are simply behavioral therapy applied to ADHD, as the hallmark features of BA relate primarily to depression. As defined in this review, however, these studies are not BA, as they seek only to modify undesirable behaviors for that end, rather than to change behavior to then affect psychological outcomes.

\section{Study designs of included studies}

The 19 relevant studies included 12 case studies/case series [45-56], three uncontrolled pre-post designs [57-59], and four RCTs [60-63].

Data from the one RCT [62] were also published in a paper focusing on mediation analyses [64], and in a paper reporting neuroimaging data [65]. All three papers were consulted for novel details for data extraction. Weersing and colleagues reported their RCT in two publications [37, 63]: data from 2017 were extracted only as these covered details from the 2016 publication. The data reported across from individuals in the case studies by Pass and colleagues [54-56] are also included in the pre/post study [59].

Table 1 provides details of study designs, participants, setting, intervention, comparator, outcome measures, and data collection points. More details in given across the online resources: Online Resource 1 details the study designs, types of difficulties addressed, and further details of participant characteristics, which are summarized here.

\section{Types of difficulties BA used to address}

BA has been used to treat depression, anxiety, and co-morbid anxiety and depression. Major depressive disorder, clinical, and sub-clinical depression have been addressed. Young people with panic, social anxiety/social phobia, generalized anxiety disorder, and/or separation anxiety have been included in the studies, with some inclusion of participants with co-morbidities amongst these difficulties.

\section{Participant characteristics}

Studies have solely been conducted in high-income settings: 13 in the USA, 4 in the UK, 1 in Japan (published across three papers), and 1 in Australia. Several of USA-based studies described ethnically diverse populations and engaging with low-income, African-American families. BA has been offered to a total of 313 adolescents [182 (58\%) female].

\section{Risk of bias}

Table 2 provides a summary of risk of bias analysis for the individual RCT studies. For just one study, an online registered protocol was available (https://clinicaltrials.gov/ct2/ show/NCT01147614), confirming no selective reporting.

Overall, risk of bias was high in relation with the challenges of blinding participants to their allocation to treatment or control group. Attrition bias between treatment and control groups was not analysed (presumably due to generally small sample sizes); therefore, it is not clear if participants' were more likely to be retained in either condition, nor if there were particular participant characteristics linked to participant attrition or incomplete data. Studies did generally use blind assessors for outcome and random sequence generation for allocation was performed in all studies.

\section{Summary of outcomes of individual studies}

Online Resource 2 provides detail of reported outcomes from all studies, together with outcome measures used, data collection points, and comparator group details, where applicable.

A range of measures have been used, with BDI and CDRS-R measures most commonly used.

Control groups received no control intervention [62]; wait-list control [60]; or referral to mental health care [37]; with just one study using an active control comparison of evidence-based intervention, being either cognitive-behavioral therapy or interpersonal therapy [61].

Although results are generally reporting data in favor of BA potentially having an effect on reducing symptoms across the disorders, the vast majority of studies are case studies and uncontrolled. 15 of the studies reported some 


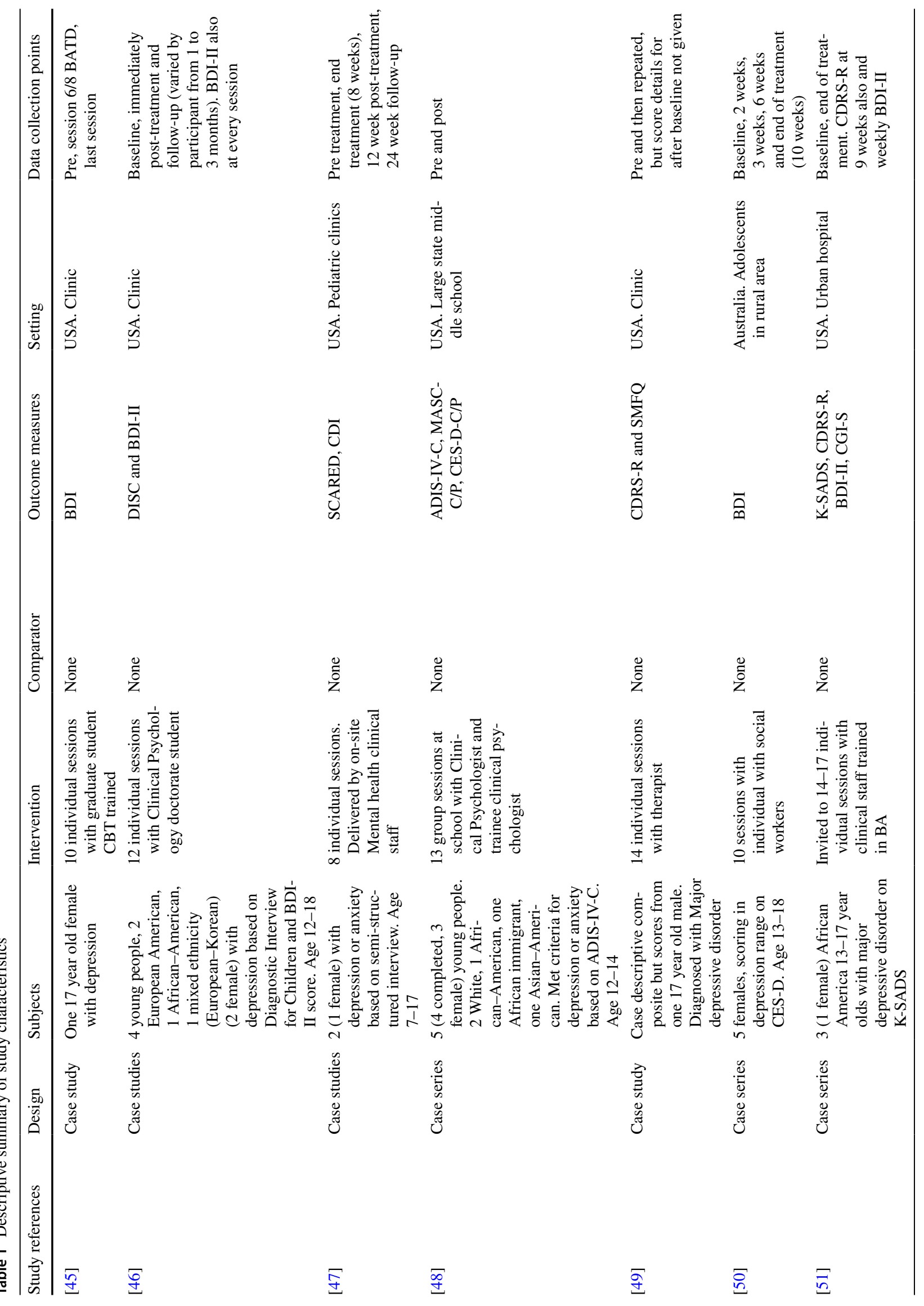




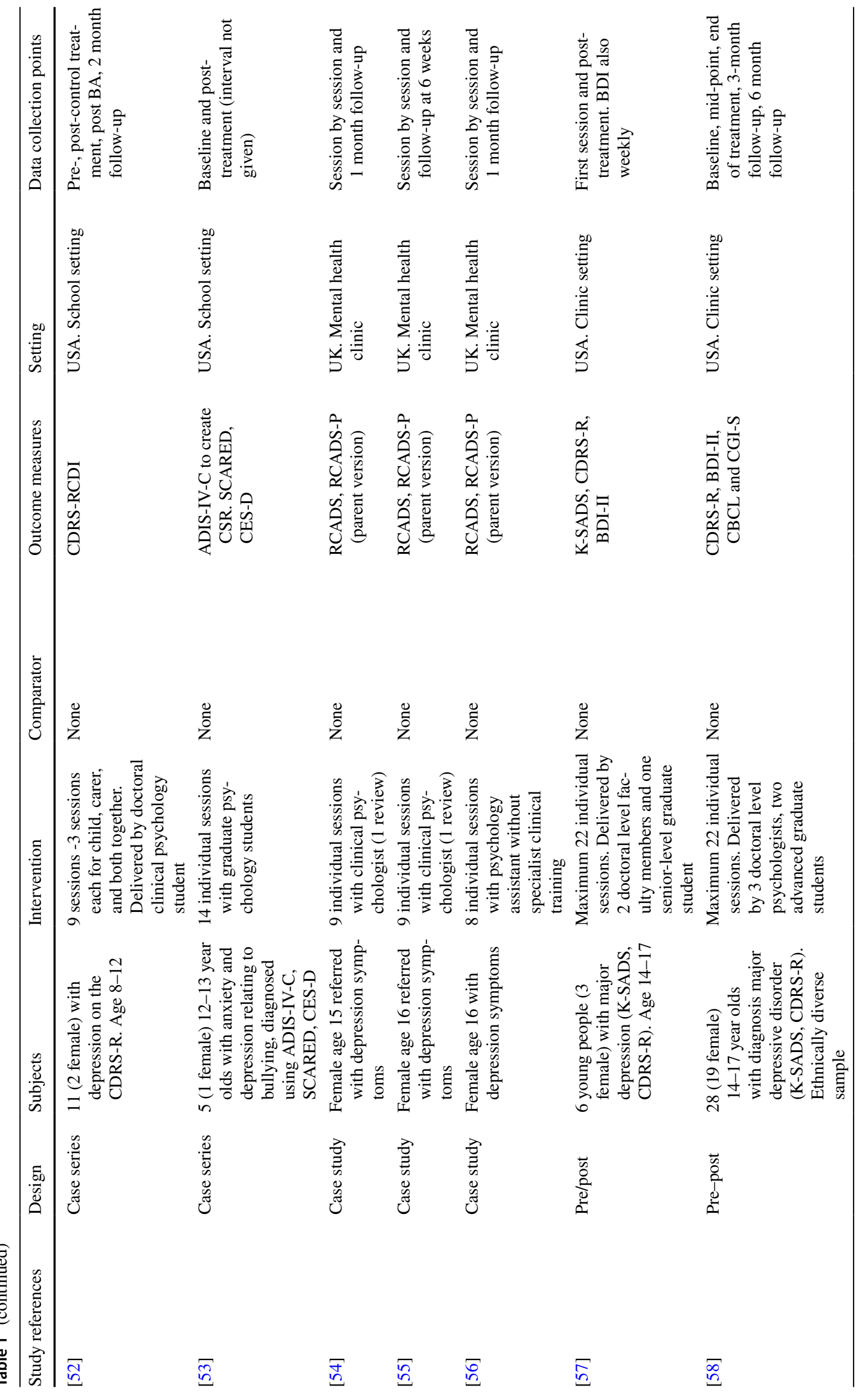




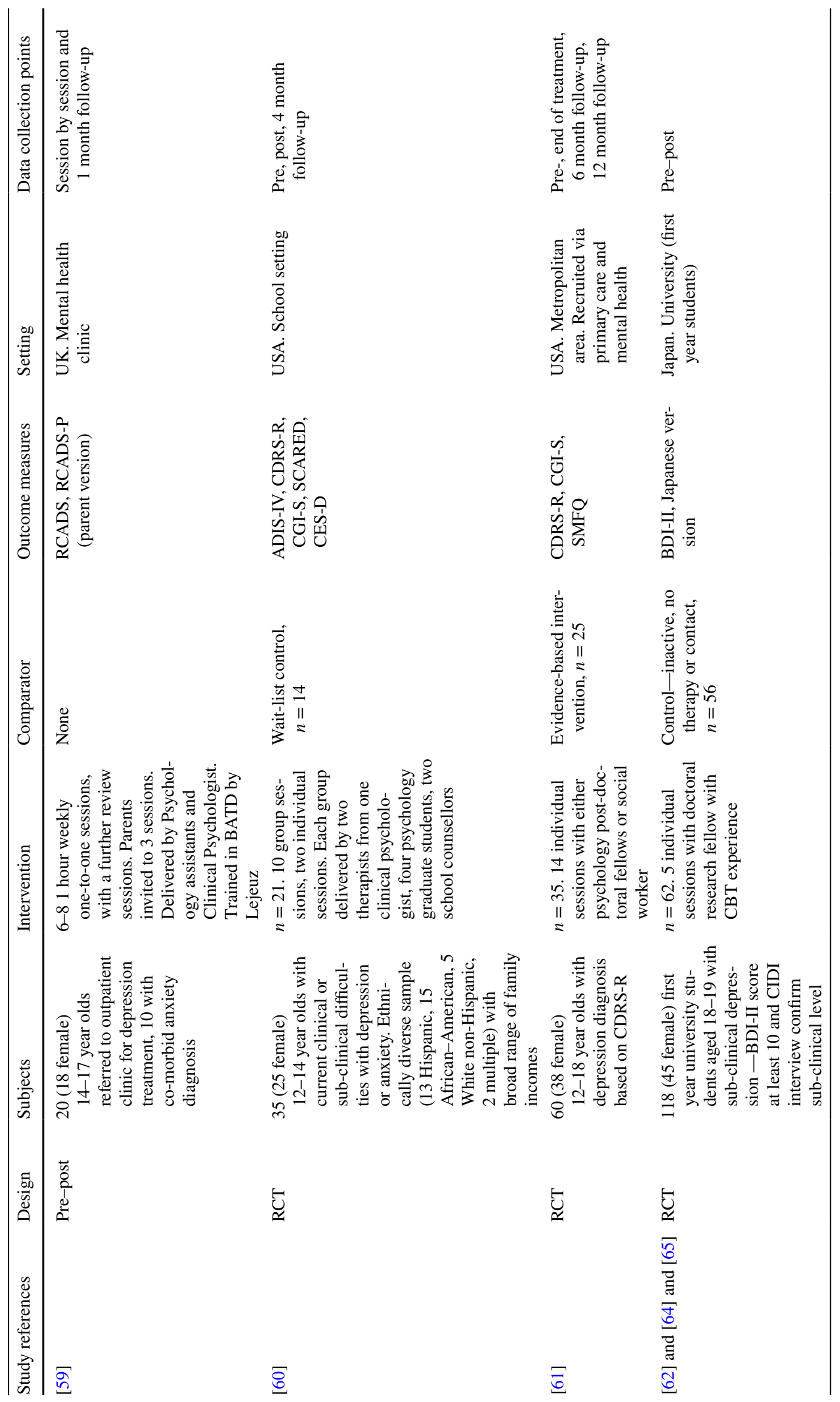




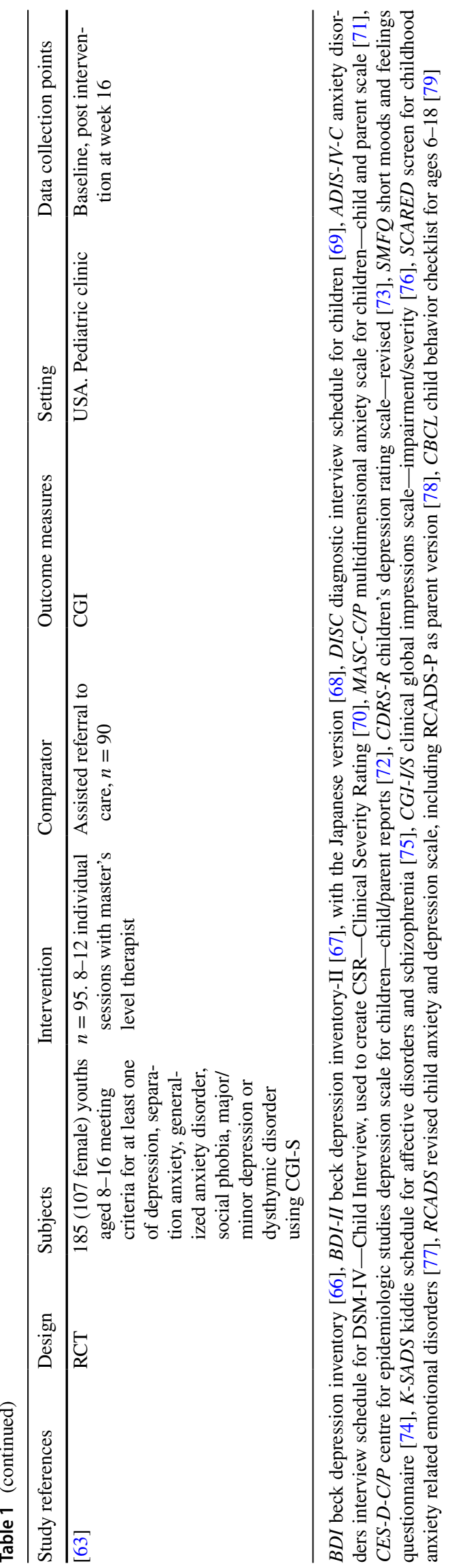

positive results (statistically significant change in severity scores or movement for majority of cases from scores indicating clinical caseness to non-caseness).

\section{Synthesis of results}

Examining only the four RCTs [37, 60-62], effect sizes (Hedges g) were calculated for each measure at post-test (not papers reported data for later follow-up periods) comparing control and BA group scores, detailed in Online Resource 3. Sample sizes are all small, with the largest study randomizing 185 people [37].

Meta-analysis was conducted with data from the RCTs. Only one RCT reported anxiety measures, as such metaanalysis focused on depression scores. Three of the four studies used the same measure (CDRS-R); therefore, data for this was measure used where possible, with the BDI scores used for the remaining study. It must be noted that these studies used different comparators: two offering no active intervention (wait-list or no intervention), one referral to mental health services, and one active treatment. The effect of BA was large, with a pooled standardized mean difference of $-0.70(95 \%$ CI $-1.20,-0.20)$ [80], see Fig. 2. This demonstrates a statistically significant difference in favor of BA; however, heterogeneity was high: $I^{2}=0.79$.

\section{BA intervention content}

Online Resource 4 details the intervention content, based on what is explicitly referred to in the studies. These categories are derived from literature reviews [19] summarizing what BA consists of (first eight columns) and common themes from the intervention descriptions. Intervention content differs in complexity, with interventions included three categories of content [52], all the way up to 10 [61]. Activity monitoring and scheduling were almost always present. Functional analysis was observed in descriptions of the interventions in 14 of the studies. Functional analysis is a wide category, including both simple analysis of antecedents and consequences of behavior in a basic three item cycle, as seen several studies using BATD, for example [45, 59]), and more complex, personalized analysis seen in BA derived from the "Washington BA" approaches [20] as seen in studies by Chu and colleagues, for example [48], using more detailed analysis of avoidance patterns and alternative responses.

\section{Details of delivery approaches for BA interventions}

Online Resource 5 summarizes location, therapist, mode, and number of sessions offered in each study. Four studies were delivered in a school setting, whilst all others were at 
Table 2 Summary of risk of bias analysis for individual studies

\begin{tabular}{lllll}
\hline Domain & $\begin{array}{l}\text { Chu et al. } \\
{[60]}\end{array}$ & $\begin{array}{l}\text { McCauley et al. } \\
{[61]}\end{array}$ & $\begin{array}{l}\text { Takagaki et al. } \\
{[62]}\end{array}$ & $\begin{array}{l}\text { Weersing } \\
\text { et al. [63] }\end{array}$ \\
\hline Random sequence generation & + & + & + & + \\
Allocation concealment & $?$ & + & + & + \\
Blinding participants and personnel & - & - & - & - \\
Blinding outcome assessment & + & + & + & + \\
Incomplete outcome data & + & + & + & + \\
Selective reporting & $?$ & $?$ & + & + \\
Other sources of bias & + & + & & + \\
\hline
\end{tabular}

Key: “+” low risk of bias, “- "high risk of bias, “?” unknown/unreported

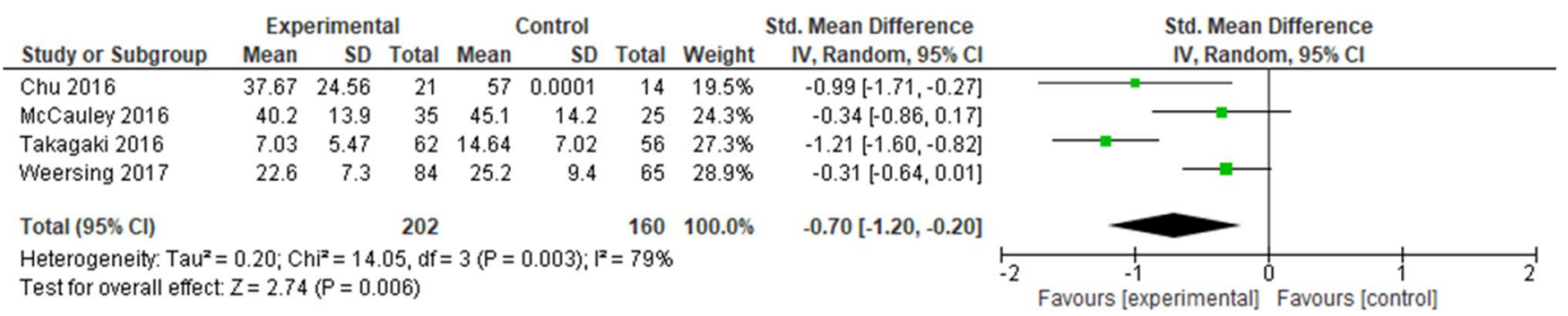

Fig. 2 Random effects meta-analysis of depression scores across included RCTs $(n=4)$

an outpatient clinic. Therapy was delivered by clinical psychologists, doctoral level psychologists, trained therapists, or social workers. Only one study used non-specialist trained staff to deliver the intervention: a psychology assistant [56]. No studies used trained lay people. All studies used faceto-face delivery of interventions, with a mixture of group and one-to-one settings (three group, one mixed group and individual, remaining 15 individual). Between five and 22 sessions were offered, and most (12 studies) descriptions included the offer of joint sessions with parents.

\section{Adaptions of BA for children and young people}

Parental involvement was the most common adaptation of BA for young people. This included parents attending sessions, workbooks for parents, and involvement of parents in supporting the young person to complete their chosen activities. Family engagement was to offer support, address barriers and create positively reinforcing interactions [47, 49, $51,54]$. This addresses also the challenges that young people have relating to how much they control their environment and how their time is spent. The wider impact that changing behavior in a young person may have on their family and other social systems needs to be considered [57].

Some studies suggest intervention with fewer techniques which are simpler to deliver and follow [37, 47]. Other studies used a greater range of techniques including skills and practice sessions to improve communication between parents and young people [45]. Problem solving skills may be particularly developmentally appropriate for young people $[49,54]$. It can be presented with acronyms to simplify and aid remembering, e.g., use of "ACTION" to present problem solving [57]. Active practice of these skills in sessions, including role play, supports learning [49]. Concrete goal setting is encouraged in one study, with awareness that must be realistic in choice of goals that young person can access resources required, for example, transport if the goal is to go to the gym [57]. It has been found useful to generate a list of developmentally appropriate activities for young people to engage in and how this could be further reinforced, including joint parent-child activities for younger children [45]. Alternatively, using developmentally appropriate prompts of life areas relevant to young people was found to help structure goal setting, e.g., "Me", "Things that matter", and "The bigger picture" [54].

Rather than structured psychoeducation, a more flexible approach is useful, for example, tailoring worksheets and handouts to the individual in a collaborative, client centered way, to maintain engagement [49, 57]. Handouts or workbooks were developed to be attractive, engaging, and developmentally appropriate and tailoring of language used to an appropriate register [47, 50, 54, 56, 57].

Homework non-completion may require attention, focusing on exploring benefits of therapy and being flexible about homework completion where needed to maintain engagement [56]. Some interventions were delivered at school, to allow easy attendance for the young person 
and use school administration systems to support intervention delivery [48].

An important adaptation is development of interventions to address both anxiety and depression, through common mechanism of reducing avoidance, as comorbidity is high $[37,47,48,53,60]$. One intervention particularly focused on the impact of the common experience of bullying on young people, tailored to ensure confidentiality was well understood by all to create an environment where they felt safe to engage in the intervention [53].

\section{Discussion}

This systematic review explored the study design, application, reach, and evidence of effectiveness for BA in children and adolescents, in addition to examining how BA has been delivered and what adaptations have been made to BA to increase suitability for application to young people.

Comparing this review with the recent systematic review and meta-analysis focusing on the effectiveness of BA for treating depression in young people [27], there are clear differences in methods that underpin the differences in reported results and discussion. The main differences in methodology are that this review did not focus specifically on depression, did not search the grey literature, did not search CINAHL (owing to lack of access to this database), but did include case studies and data extraction extended to more detailed descriptions of intervention delivery, content, and adaptations. This reflects the differences in the two reviews' objectives. This review then included studies not only focusing on depression but also on anxiety and did not include two studies seen in Tindall's review that are not published in peer-reviewed journals [81, 82].

Research into BA with young people is at an early stage, reflected in study numbers, design, and quality. The reach of the research to date is limited. Although depression and a range of anxiety disorders have been included, the sample size and diversity of young people involved in the research remain very limited. Included studies do not cover children and adolescents with medical problems, where rates depression and anxiety are higher than in a physically healthy population [83]. Despite growing acknowledgement of the importance of young people's mental health globally [1], studies have all taken place in high-income countries. Whilst this is not surprising in terms of access to funding for early stage research, it is now important to explore how this intervention could be applied in other contexts. It is not clear to what extent BA is feasible in situations of poverty and impoverished environments, where details of and access to positively reinforcing activities may be very different, and in cultures were the identity, social development, and role of children and young people may differ.
The majority of studies are case studies, which have more recently informed RCTs. As such, it is not possible to draw firm conclusions regarding the effectiveness of BA to treat child and adolescent mental health problems. However, the data reported are typically promising as to the intervention's potential effect and meta-analysis for depression indicated effect in favor of BA, although with some methodological limitations. The feasibility of this approach with this age group is supported.

The review found that BA has been delivered by at least graduate level psychologists if not fully qualified therapists or health and social care workers. BA has been delivered to adults by lay staff, increasing its potential scalability [84]. Meeting the demand for effective therapies for children and adolescents is a priority, yet nothing is known currently about the use of lay therapist in BA's delivery to this group. Given that many of the adaptations made to BA revealed in this review are to make it flexible in delivery to engage young people, it is not clear whether lay delivery would be possible, or if a higher level of technical skill will be required to tailor treatments to children and adolescents.

BA has been primarily recruited to and delivered through mental health and education systems, with just three studies recruiting via general community routes [46, 57, 58]. Many young people with mental health problems may be disengaged from education and not joined up with mental health services [10, 12, 85]. Implementing BA outside of existing systems may present greater challenges to its feasibility, in terms of recruitment and retention but also in relation with BA mechanisms. Based on increased positive reinforcement from activities, BA requires that the participant can access and engage in such activities. Availability of social activities, for example, may be scant for marginalized or impoverished young people. To address implementation challenges, researchers may need to explore and intervene with what activities are actually available in the wider environment and perhaps include in the overall intervention plan development or connections to community youth groups, for example.

What has been delivered as "BA" and how it was delivered are varied. Some descriptions of intervention content are poor in detail and quality. Despite several studies reportedly using the same intervention, descriptions of the interventions vary-for example across the studies conducted by Chu et al., only one makes specific reference to including relapse prevention [60]. This difficulty in reporting is well acknowledged [86], and researchers in behavior change interventions have developed taxonomies to attempt to improve descriptions [87]. A similar approach for mental health interventions may be of use, to encourage authors to clearly and fully describe the essential techniques used in their interventions and to allow readers and systematic reviewers to better classify intervention content. 
The optimum content of BA for this population remains unclear. "Functional analysis" content is seen in many interventions reviewed here, but varies significantly in its emphasis, depth, and complexity. The inclusion of functional analysis, explicit addressing of avoidance, and targeting of verbal behaviors, for example, may or may not improve outcomes. This issue is also seen in the BA for adults' literature; for example, whilst avoidance is explicitly targeted in several BA interventions, there is little direct support for this over and above activity scheduling [19]. A review, with sub-group analysis, of the adult BA literature concluded that there appeared to be no impact on outcome as to whether in-depth functional analysis was included or not [5]. As research develops, optimizing the intervention to be efficient and cost-effective will be necessary.

Adaptations made to BA to use with young people have revealed themes in ensuring flexibility of delivery, partly to maintain engagement, and designing tailored materials and lists of activities that are developmentally appropriate. BA is found to be feasible with children and adolescents in terms of the cognitive demands and developmental appropriacy: participants could follow materials, generate lists of goals, and engage in the techniques offered.

Parental involvement is a key issue in interventions with children and adolescents. Overall, inconsistent support has been found for parental involvement in relation with cognitive-behavioral therapy outcomes [88]. This review highlights its importance to allow the young person to meaningfully engage in therapy through being able to carry out scheduled activities with parental support and resources, where needed. The necessity of this will vary by individual's context and research could develop brief, clear protocols to assess if/what parental involvement may be required for each young person. Such protocols may be essential for delivery of BA by non-specialists. Given the direct interaction with the environment that BA demands, there may be a ceiling of change where parents, and wider social environments are not taken into account: BA may be able to impact positively, but reach a ceiling where the young person cannot do planned activities, or continues to experience conflict in the home that relates to presenting the depression or anxiety. Indeed, barriers and facilitators to implementation of BA for children and adolescents have received little attention in the literature to date.

\section{Limitations}

This review included only published studies, studies with at least basic reporting of outcomes data and studies focusing on treating, rather than preventing, mental health difficulties. We did not contact authors to obtain further unpublished information. The extent of publication bias towards positive results is unknown.
Three excluded studies described BA style interventions, but did not provide any participant outcome data. Spirito et al. used BA techniques with children with cystic fibrosis to address anxiety [36]. Davidson et al. explored the use of web-based BA with adolescents with depression [34]. Welsh et al. discussed the potential application of BA for adolescents at risk of psychosis [35]. These areas of clinical health, online delivery, and psychosis represent further potential areas of development for BA research. This review does not include any qualitative findings from work with these client groups.

The methodological limitations of the studies reflect the early stage of research into BA for this population. Case studies provide little information regarding effectiveness; however, these reports together with the pre/post and RCT reports offer detailed information regarding the design of the intervention itself.

Meta-analysis was completed; however, results must be interpreted with caution owing to high heterogeneity, risk of bias, differences in control conditions, and one study used a different depression outcome. Risk of bias was particularly relevant in relation with the general lack of published protocols, meaning that it is not possible to evaluate reporting bias. Furthermore, owing to the nature of the research, concealment of allocation from participants and those delivering the intervention was problematic in the RCTs, particularly those that did not use an active intervention in the control group. Meta-analysis was not possible for the application of BA to anxiety for young people, as only one RCT included this outcome.

\section{Conclusions}

This review provides a detailed examination of the state of the science and details of intervention content, to inform future work into intervention design and methodology of future studies. Whilst there is support for the feasibility of the intervention, and substantial recommendations for how this can be delivered, there is insufficient evidence to draw conclusions regarding effectiveness, although initial data support effectiveness for depression. Recommendations for future research include the need to develop and test BA for young people in low- and middle-income countries and with a range of cultures, paying attention to how BA may need to be further adapted for young people where their social development and role may differ to those in high-income countries. Methodologically, it is now necessary to move to fully powered RCTs to explore effectiveness in detail. Alongside this, issues of how to optimize intervention content to ensure maximum efficiency and cost-effectiveness must be considered, to aid scalability. 
To extend the potential practical promise and implementation of BA, it is important to explore issues such as BA can be effectively delivered to children and adolescents using lay therapists and to document and address barriers and facilitators to implementation, alongside effectiveness research.

\section{Compliance with ethical standards}

Conflict of interest The authors declare that they have no conflict of interest.

Open Access This article is distributed under the terms of the Creative Commons Attribution 4.0 International License (http://creativeco mmons.org/licenses/by/4.0/), which permits unrestricted use, distribution, and reproduction in any medium, provided you give appropriate credit to the original author(s) and the source, provide a link to the Creative Commons license, and indicate if changes were made.

\section{References}

1. Patton GC, Sawyer SM, Santelli JS, Ross DA, Afifi R, Allen NB, Arora M, Azzopardi P, Baldwin W, Bonell C, Kakuma R, Kennedy E, Mahon J, McGovern T, Mokdad AH, Patel V, Petroni S, Reavley N, Taiwo K, Waldfogel J, Wickremarathne D, Barroso C, Bhutta Z, Fatusi AO, Mattoo A, Diers J, Fang J, Ferguson J, Ssewamala F, Viner RM (2016) Our future: a Lancet commission on adolescent health and wellbeing. Lancet. https://doi.org/10.1016/ S0140-6736(16)00579-1

2. Erskine H, Moffitt T, Copeland W, Costello E, Ferrari A, Patton G, Degenhardt L, Vos T, Whiteford H, Scott J (2015) A heavy burden on young minds: the global burden of mental and substance use disorders in children and youth. Psychol Med 45(07):1551-1563

3. Baxter A, Vos T, Scott K, Ferrari A, Whiteford H (2014) The global burden of anxiety disorders in 2010. Psychol Med 44(11):2363-2374

4. Garber J, Weersing VR (2010) Comorbidity of anxiety and depression in youth: implications for treatment and prevention. Clin Psychol Sci Pract 17(4):293-306

5. Ekers D, Webster L, Van Straten A, Cuijpers P, Richards D, Gilbody S (2014) Behavioural activation for depression; an update of meta-analysis of effectiveness and sub group analysis. PLOS ONE 9(6):e100100

6. Richards DA, Ekers D, McMillan D, Taylor RS, Byford S, Warren FC, Barrett B, Farrand PA, Gilbody S, Kuyken W, O'Mahen H, Watkins ER, Wright KA, Hollon SD, Reed N, Rhodes S, Fletcher E, Finning K (2016) Cost and outcome of behavioural activation versus cognitive behavioural therapy for depression (COBRA): a randomised, controlled, non-inferiority trial. Lancet 388(10047):871-880. https://doi.org/10.1016/S0140 -6736(16)31140-0

7. Das JK, Salam RA, Lassi ZS, Khan MN, Mahmood W, Patel V, Bhutta ZA (2016) Interventions for adolescent mental health: an overview of systematic reviews. J Adolesc Health 59(4 Supplement):49-60. https://doi.org/10.1016/j.jadohealth.2016.06.020

8. Creswell C, Waite P, Cooper PJ (2014) Assessment and management of anxiety disorders in children and adolescents. Arch Dis Child. https://doi.org/10.1136/archdischild-2013-303768

9. NICE (2015) CG28 depression in children and young people: identification and management. National Institute Clinical Excellence, London
10. Knapp M, Ardino V, Brimblecombe N, Evans-Lacko S, Lemmi V, King D, Snell T (2016) Youth mental health: new economic evidence. Personal social services research unit. http://www.pssru .ac.uk/archive/pdf/5160.pdf. Accessed on April 2017

11. Kovess-Masfety V, Van Engelen J, Stone L, Otten R, Carta MG, Bitfoi A, Koc C, Goelitz D, Lesinskiene S, Mihova Z (2017) Unmet need for specialty mental health services among children across Europe. Psychiatr Serv 68(8):789-795

12. Patel V, Flisher AJ, Nikapota A, Malhotra S (2008) Promoting child and adolescent mental health in low and middle income countries. J Child Psychol Psychiatry 49(3):313-334

13. Dimidjian S, McCauley E (2016) Modular, scalable, and personalized: priorities for behavioral interventions for adolescent depression. Clin Psychol Sci Pract 23(1):58-61

14. Fernando S (2014) Mental health worldwide: culture, globalization and development. Macmillan, Palgrave

15. Lund C, Tomlinson M, De Silva M, Fekadu A, Shidhaye R, Jordans M, Petersen I, Bhana A, Kigozi F, Prince M, Thornicroft G, Hanlon C, Kakuma R, McDaid D, Saxena S, Chisholm D, Raja S, Kippen-Wood S, Honikman S, Fairall L, Patel V (2012) PRIME: a programme to reduce the treatment gap for mental disorders in five low- and middle-income countries. PLoS Med 9(12):e1001359. https://doi.org/10.1371/journal.pmed.1001359

16. Dimidjian S, Hollon SD, Dobson KS, Schmaling KB, Kohlenberg RJ, Addis ME, Gallop R, McGlinchey JB, Markley DK, Gollan JK (2006) Randomized trial of behavioral activation, cognitive therapy, and antidepressant medication in the acute treatment of adults with major depression. J Consul Clin Psychol 74(4):658

17. Kanter J, Puspitasari AJ, Santos MM, Nagy GA (2012) Behavioural activation: history, evidence and promise. Br J Psychiatry 200(5):361-363

18. Ekers D, Richards D, McMillan D, Bland JM, Gilbody S (2011) Behavioural activation delivered by the non-specialist: phase II randomised controlled trial. Br J Psychiatry 198(1):66-72. https ://doi.org/10.1192/bjp.bp.110.079111

19. Kanter JW, Manos RC, Bowe WM, Baruch DE, Busch AM, Rusch LC (2010) What is behavioral activation? a review of the empirical literature. Clin Psychol Rev 30(6):608-620. https://doi. org/10.1016/j.cpr.2010.04.001

20. Martell CR, Addis ME, Jacobson NS (2001) Depression in context: strategies for guided action. WW Norton \& Co, New York

21. Lejuez C, Hopko DR, LePage JP, Hopko SD, McNeil DW (2001) A brief behavioral activation treatment for depression. Cogn Behav Pract 8(2):164-175

22. Dimidjian S, Barrera M Jr, Martell C, Muñoz RF, Lewinsohn PM (2011) The origins and current status of behavioral activation treatments for depression. Ann Rev Clin Psychol 7:1-38

23. Hopko DR, Lejuez CW, Ryba MM, Shorter RL, Bell JL (2016) Support for the efficacy of behavioural activation in treating anxiety in breast cancer patients. Clin Psychol 20(1):17-26

24. Chen J, Liu X, Rapee RM, Pillay P (2013) Behavioural activation: a pilot trial of transdiagnostic treatment for excessive worry. Behav Res Ther 51(9):533-539. https://doi.org/10.1016/j. brat.2013.05.010

25. Hopko DR, Robertson S, Lejuez C (2006) Behavioral activation for anxiety disorders. Behav Anal Today 7(2):212

26. LeBlanc LA, Gillis JM (2012) Behavioral interventions for children with autism spectrum disorders. Pediatr Clin N Am 59(1):147-156. https://doi.org/10.1016/j.pcl.2011.10.006

27. Tindall L, Mikocka Walus A, McMillan D, Wright B, Hewitt C, Gascoyne S (2017) Is behavioural activation effective in the treatment of depression in young people? a systematic review and meta analysis. Psychol Psychother Theor Res Pract 90(4):770-796 
28. Leon AC, Davis LL, Kraemer HC (2011) The role and interpretation of pilot studies in clinical research. J Psychiatr Res 45(5):626-629

29. Fletcher A, Jamal F, Moore G, Evans RE, Murphy S, Bonell C (2016) Realist complex intervention science: applying realist principles across all phases of the medical research council framework for developing and evaluating complex interventions. Evaluation 22(3):286-303

30. WHO (2015) Adolescent Health Research Priorities: Report of a Technical Consultation. vol Accessed online March 2016 from http://apps.who.int/iris/bitstream/10665/203564/1/WHO_FWC_ MCA_15_07_eng.pdf?ua=1. World Health Organization (WHO), Geneva

31. Higgins JPT, Green S (eds) (2008) Cochrane handbook for systematic review of interventions. Wiley, Chicester

32. Higgins JP, Altman DG, Gøtzsche PC, Jüni P, Moher D, Oxman AD, Savović J, Schulz KF, Weeks L, Sterne JA (2011) The cochrane collaboration's tool for assessing risk of bias in randomised trials. BMJ 343:d5928

33. Moher D, Liberati A, Tetzlaff J, Altman DG (2010) Preferred reporting items for systematic reviews and meta-analyses: the PRISMA statement. Int J Surg 8(5):336-341. https://doi. org/10.1016/j.ijsu.2010.02.007

34. Davidson TM, Yuen EK, Felton JW, McCauley J, Gros KS, Ruggiero KJ (2014) Feasibility assessment of a brief, web-based behavioral activation intervention for adolescents with depressed mood. Int J Psychiatry Med 48(1):69-82

35. Welsh P, Kitchen CEW, Ekers D, Webster L, Tiffin PA (2016) Behavioural activation therapy for adolescents 'at risk' for psychosis? Early Intervention in Psychiatry 10(2):186-188

36. Spirito A, Russo DC, Masek BJ (1984) Behavioral interventions and stress management training for hospitalized adolescents and young adults with cystic fibrosis. Gen Hosp Psychiatry 6(3):211218. https://doi.org/10.1016/0163-8343(84)90042-2

37. Weersing VR, Rozenman M, Gonzalez A, Jeffreys M, Porta G, Brent DA (2016) Brief behavioral therapy for pediatric anxiety and depression in primary care: a randomized clinical trial. J Am Acad Child Adolesc Psychiatry 55(10):S336. https://doi. org/10.1016/j.jaac.2016.07.406

38. MacPherson L, Collado A, Ninnemann A, Hoffman E (2017) Development of a behavioral activation-based intervention for cigarette-smoking young adults. Cogn Behav Pract 24(1):101114. https://doi.org/10.1016/j.cbpra.2016.03.004

39. Chu BC, Merson RA, Zandberg LJ, Areizaga M (2012) Calibrating for comorbidity: clinical decision-making in youth depression and anxiety. Cogn Behav Pract 19(1):5-16

40. Manglick M, Rajaratnam SM, Taffe J, Tonge B, Melvin G (2013) Persistent sleep disturbance is associated with treatment response in adolescents with depression. Aus N Zeal J Psychiatry 47(6):556-563. https://doi.org/10.1177/0004867413481630

41. Curtis DF (2010) ADHD symptom severity following participation in a pilot, 10-week, manualized, family-based behavioral intervention. Child Fam Behav Ther 32(3):231-241. https://doi. org/10.1080/07317107.2010.500526

42. Curtis DF, Chapman S, Dempsey J, Mire S (2013) Classroom changes in ADHD symptoms following clinic-based behavior therapy. J Clin Psychol Med Settings 20(1):114-122

43. Rudd MD, Rajab MH, Orman DT, Joiner T, Stulman DA, Dixon W (1996) Effectiveness of an outpatient intervention targeting suicidal young adults: preliminary results. J Consult Clin Psychol 64(1):179-190. https://doi.org/10.1037/0022-006X.64.1.179

44. Harper JM, Iwata BA, Camp EM (2013) Assessment and treatment of social avoidance. J Appl Behav Anal 46(1):147-160

45. Ruggiero KJ, Morris TL, Hopko DR, Lejuez CW (2007) Application of behavioral activation treatment for depression to an adolescent with a history of child maltreatment. Clin Case Stud 6(1):64-78

46. Gaynor ST, Harris A (2008) Single-participant assessment of treatment mediators: strategy description and examples from a behavioral activation intervention for depressed adolescents. Behav Modif 32(3):372-402

47. Weersing VR, Gonzalez A, Campo JV, Lucas AN (2008) Brief behavioral therapy for pediatric anxiety and depression: piloting an integrated treatment approach. vol 15. Assoc Adv Behav Ther. https://doi.org/10.1016/j.cbpra.2007.10.001

48. Chu BC, Colognori D, Weissman AS, Bannon K (2009) An initial description and pilot of group behavioral activation therapy for anxious and depressed youth. Cogn Behav Pract 16(4):408-419

49. McCauley E, Schloredt K, Gudmundsen G, Martell C, Dimidjian $S$ (2011) Expanding behavioral activation to depressed adolescents: lessons learned in treatment development. Cogn Behav Pract 18(3):371-383

50. Wallis A, Roeger L, Milan S, Walmsley C, Allison S (2012) Behavioural activation for the treatment of rural adolescents with depression. Aus J Rural Health 20(2):95-96

51. Jacob M, Keeley ML, Ritschel L, Craighead WE (2013) Behavioural activation for the treatment of low-income, African American adolescents with major depressive disorder: a case series. Clin Psychol Psychother 20(1):87-96

52. Riley AR, Gaynor ST (2014) Identifying mechanisms of change: Utilizing single-participant methodology to better understand behavior therapy for child depression. Behav Modif 38(5):636-664

53. Chu BC, Hoffman L, Johns A, Reyes-Portillo J, Hansford A (2015) Transdiagnostic behavior therapy for bullying-related anxiety and depression: initial development and pilot study. Cogn Behav Pract 22(4):415-429

54. Pass L, Brisco G, Reynolds S (2015) Adapting brief behavioural activation (BA) for adolescent depression: a case example. Cogn Behav Ther 8:e17

55. Pass L, Whitney H, Reynolds S (2016) Brief behavioral activation for adolescent depression: working with complexity and risk. Clin Case Stud 15(5):360-375

56. Pass L, Hodgson E, Whitney H, Reynolds S (2017) Brief behavioral activation treatment for depressed adolescents delivered by nonspecialist clinicians: a case illustration. Cogn Behav Pract. https://doi.org/10.1016/j.cbpra.2017.05.003

57. Ritschel LA, Ramirez CL, Jones M, Craighead W (2011) Behavioral activation for depressed teens: a pilot study. Cogn Behav Pract 18(2):281-299

58. Ritschel LA, Ramirez CL, Cooley JL, Craighead W (2016) Behavioral activation for major depression in adolescents: results from a pilot study. Clin Psychol Sci Pract 23(1):39-57

59. Pass L, Lejuez CW, Reynolds S (2017) Brief behavioural activation (Brief BA) for adolescent depression: A pilot study. Behav Cogn Psychother 1-13. https://doi.org/10.1017/s13524658170004 43

60. Chu BC, Crocco ST, Esseling P, Areizaga MJ, Lindner AM, Skriner LC (2016) Transdiagnostic group behavioral activation and exposure therapy for youth anxiety and depression: initial randomized controlled trial. Behav Res Ther 76:65-75

61. McCauley E, Gudmundsen G, Schloredt K, Martell C, Rhew I, Hubley S, Dimidjian S (2016) The adolescent behavioral activation program: adapting behavioral activation as a treatment for depression in adolescence. J Clin Child Adolesc Psychol 45(3):291-304

62. Takagaki K, Okamoto Y, Jinnin R, Mori A, Nishiyama Y, Yamamura T, Yokoyama S, Shiota S, Okamoto Y, Miyake Y, Ogata A, Kunisato Y, Shimoda H, Kawakami N, Furukawa TA, Yamawaki S (2016) Behavioral activation for late adolescents with 
subthreshold depression: A randomized controlled trial. Eur Child Adolesc Psychiatry 25(11):1171-1182

63. Weersing VR, Brent DA, Rozenman MS, Gonzalez A, Jeffreys M, Dickerson JF, Lynch FL, Porta G, Iyengar S (2017) Brief behavioral therapy for pediatric anxiety and depression in primary care: a randomized clinical trial. JAMA Psychiatry 74(6):571-578. https ://doi.org/10.1001/jamapsychiatry.2017.0429

64. Takagaki K, Jinnin R, Mori A, Nishiyama Y, Yamamura T, Yokoyama S, Shiota S, Okamoto Y, Miyake Y, Ogata A, Shimoda H, Kawakami N, Furukawa TA, Yamawaki S (2016) Mechanisms of behavioral activation for late adolescents: positive reinforcement mediate the relationship between activation and depressive symptoms from pre-treatment to post-treatment. J Affect Disord 204:70-73

65. Mori A, Okamoto Y, Okada G, Takagaki K, Jinnin R, Takamura M, Kobayakawa M, Yamawaki S (2016) Behavioral activation can normalize neural hypoactivation in subthreshold depression during a monetary incentive delay task. J Affect Disord 189:254-262

66. Beck AT, Ward CH, Mendelson M, Mock J, Erbaugh J (1961) An inventory for measuring depression. Arch Gen Psychiatry 4(6):561-571

67. Beck AT, Steer RA, Brown GK (1996) Beck depression inventoryII. San Antonio 78(2):490-498

68. Kojima M, Furukawa TA (2003) Japanese version of the beck depression inventory. Nippon-Hyoron-sha Co., Tokyo

69. Shaffer D, Fisher P, Lucas CP, Dulcan MK, Schwab-Stone ME (2000) NIMH diagnostic interview schedule for children version IV (NIMH DISC-IV): description, differences from previous versions, and reliability of some common diagnoses. J Am Acad Child Adolesc Psychiatry 39(1):28-38

70. Silverman WK, Albano AM (1996) Anxiety disorders interview schedule for DSM-IV: child interview schedule, vol 2. Graywind Publications

71. March JS, Parker JD, Sullivan K, Stallings P, Conners CK (1997) The multidimensional anxiety scale for children (MASC): factor structure, reliability, and validity. J Am Acad Child Adolesc Psychiatry 36(4):554-565

72. Radloff LS (1977) The CES-D scale: a self-report depression scale for research in the general population. Appl Psychol Meas 1(3):385-401

73. Poznanski EO, Mokros HB (1996) Children's depression rating scale, revised (CDRS-R). Western Psychological Services, Los Angeles

74. Angold A, Costello EJ, Messer SC, Pickles A (1995) Development of a short questionnaire for use in epidemiological studies of depression in children and adolescents. Int J Methods Psychiatr Res

75. Kaufman J, Birmaher B, Brent D, Rao U, Flynn C, Moreci P, Williamson D, Ryan N (1997) Schedule for affective disorders and schizophrenia for school-age children-present and lifetime version (K-SADS-PL): initial reliability and validity data. J Am Acad Child Adolesc Psychiatry 36(7):980-988

76. Rapoport J, Conners C (1985) Rating scales and assessment instruments for use in pediatric psychopharmacology research-introduction. National Institute of Mental Health, Washington

77. Birmaher B, Khetarpal S, Brent D, Cully M, Balach L, Kaufman J, Neer SM (1997) The screen for child anxiety related emotional disorders (SCARED): scale construction and psychometric characteristics. J Am Acad Child Adolesc Psychiatry 36(4):545-553

78. Chorpita BF, Yim L, Moffitt C, Umemoto LA, Francis SE (2000) Assessment of symptoms of DSM-IV anxiety and depression in children: a revised child anxiety and depression scale. Behav Res Ther 38(8):835-855

79. Achenbach T, Rescorla L (2001) Manual for the Achenbach system of empirically based assessment school-age forms profiles. Aseba, Burlington

80. Cohen J (1988) Statistical power analysis for the behavioral sciences, 2nd edn. Lawrence Erlbaum Associates Inc, New Jersey

81. Douleh TN (2014) Motivational interviewing assessment and behavior therapy as a stepped-care approach to the treatment of adolescent depression. Dissertation Abstracts International: section B: The Sciences and Engineering 74 (11-B(E)): No Pagination Specified

82. Stark KD (1985) A comparison of the relative efficacy of self-control therapy and behaviour therapy for the reduction of depression in children. University of Wisconsin, Madison, WI. Unpublished doctoral dissertation

83. Thabrew H, Stasiak K, Hetrick SE, Wong S, Huss JH, Merry SN (2017) Psychological therapies for anxiety and depression in children and adolescents with longterm physical conditions. Accessed online June 2017 from http://onlinelibrary.wiley.com/ doi/10.1002/14651858.CD012488/full, The Cochrane Library

84. Singla DR, Weobong B, Nadkarni A, Chowdhary N, Shinde S, Anand A, Fairburn CG, Dimijdan S, Velleman R, Weiss H, Patel V (2014) Improving the scalability of psychological treatments in developing countries: an evaluation of peer-led therapy quality assessment in Goa, India. Behav Res Ther 60(100):53-59. https ://doi.org/10.1016/j.brat.2014.06.006

85. Kieling C, Baker-Henningham H, Belfer M, Conti G, Ertem I, Omigbodun O, Rohde LA, Srinath S, Ulkuer N, Rahman A (2011) Child and adolescent mental health worldwide: evidence for action. The Lancet 378(9801):1515-1525

86. Michie S, Fixsen D, Grimshaw J, Eccles M (2009) Specifying and reporting complex behaviour change interventions: the need for a scientific method. Implement Sci 4(1):40. https://doi. org/10.1186/1748-5908-4-40

87. Michie S, Richardson M, Johnston M, Abraham C, Francis J, Hardeman W, Eccles MP, Cane J, Wood CE (2013) The behavior change technique taxonomy (v1) of 93 hierarchically clustered techniques: building an international consensus for the reporting of behavior change interventions. Ann Behav Med 46(1):81-95. https://doi.org/10.1007/s12160-013-9486-6

88. Breinholst S, Esbjørn BH, Reinholdt-Dunne ML, Stallard P (2012) CBT for the treatment of child anxiety disorders: a review of why parental involvement has not enhanced outcomes. J Anxiety Disord 26(3):416-424. https://doi.org/10.1016/j.janxdis.2011.12.014 